\title{
Nurse-targeted care for HIV positive persons with CD4<100 improved time to ART initiation and retention in Uganda
}

\author{
Agnes N Kiragga ${ }^{1 *}$, Elizabeth Nalintya ${ }^{1}$, Bozena Morawski ${ }^{2}$ Joanita Kigozi ${ }^{1}$, Benjamin J Park , Jonathan E Kaplan³, \\ David R.Boulware ${ }^{2}$, David B Meya ${ }^{1,2,4}$, Yukari C Manabe ${ }^{5}$
}

From 7th Annual Conference on the Science of Dissemination and Implementation in Health North Bethesda, MD, USA. 8-9 December 2014

\section{Background}

HIV testing and retention in care treatment are key strategies towards an AIDS free generation. Immediate treament of HIV patients with the lowest CD4 T cell counts is critical given their high rates of pre-antiretroviral therapy mortality. We evaluated the impact of adding an additional nurse-counsellor on HIV outcomes among patients enrolled in care in 7 urban public clinics in Kampala, Uganda.

\section{Methods}

One additional research nurse per clinic was specifically tasked with identifying and tracking HIV-infected persons with $\mathrm{CD} 4<100$ cells $/ \mu \mathrm{L}$ who were lost, and expediting ART initiation among patients enrolled after July 2012. Data were also retrospectively collected on all patients with $\mathrm{CD} 4<100$ cells $/ \mu \mathrm{L}$ that registered at these clinics in the latter six months of 2011. We compared time from CD4 blood draw to ART initiation, frequency of CD4 testing and 6 month retention-in-care by time period. Analyses of categorical and continuous variables were conducted using X2 and Mann-Whitney tests.

\section{Results}

A total of 258 patients in the 2011 cohort and 593 in the 2012 cohort completed 6 months from clinic registration. Median age of patients in both cohorts was 32 years and $55 \%$ were female. Median CD 4 cells/ $\mu \mathrm{L}$ [Interquartile, IQR] count in the 2011 cohort was 34 [13, 61] and 43 $[19,71]$ in $2012(\mathrm{p}<0.002)$. Median days from CD4 blood draw to ART initiation reduced from 42 [IQR: 28, 56] in the 2011 to 33 [IQR: 21, 47] in 2012, p < 0.001. Six-month retention in care was $62.0 \%(160 / 258)$ in 2011 cohort compared to $75.9 \%(450 / 593)$ in 2012 ( $\mathrm{p}<0.001)$. Among the patients retained in care, 24.4\% (39/160) received a CD4 count test at 6 months versus 53.3\% $(240 / 450)$ in the 2012 cohort.

\section{Conclusion}

The addition of one nurse per clinic to identify and follow severely immune-compromised new clinic patients with $\mathrm{CD} 4<100$ led to improved patient care and better outcomes. Funder: The study is an Implementation Science project funded by CDC.

\section{Authors' details}

'Infectious Diseases Institute, College of Health Sciences, Makerere University, Kampala, 256, Uganda. ${ }^{2}$ Division of Infectious Disease and International Medicine, Department of Medicine, University of Minnesota, Minneapolis, MN, 55455, USA. ${ }^{3}$ Division of Global HIV/AIDS (DGHA), Center for Global Health (CGH), Centers for Disease Control and Prevention, Atlanta, GA, 30333, USA. ${ }^{4}$ School of Medicine, College of Health Sciences, Makerere University, Kampala, 256, Uganda. ${ }^{5}$ Division of Infectious Diseases, Department of Medicine, Johns Hopkins University School of Medicine, Baltimore, MD, 21205, USA.

Published: 20 August 2015

doi:10.1186/1748-5908-10-S1-A81

Cite this article as: Kiragga et al: Nurse-targeted care for HIV positive persons with CD4<100 improved time to ART initiation and retention in Uganda. Implementation Science 2015 10(Suppl 1):A81.

\footnotetext{
* Correspondence: agnes.kiragga@gmail.com

'Infectious Diseases Institute, College of Health Sciences, Makerere University, Kampala, 256, Uganda

Full list of author information is available at the end of the article
} 